\title{
Some Discussions on The Establishment of a Scientific Cosmological Model
}

\author{
Weicheng Cui ${ }^{1,2^{*}}$ \\ ${ }^{1}$ Westlake University, China \\ ${ }^{2}$ Shanghai Ocean University, China
}

Submission: July 06, 2019; Published: July 24, 2019

"Corresponding author: Weicheng Cui, Chair professor at Westlake University, China and adjunct professor at Shanghai Ocean University, Shanghai 201306, China

\begin{abstract}
The Big Bang theory is the currently most prevailing cosmological model for the observable universe, but it still exists many un-answered questions such as the existence of the Singularity and the reason for a sudden explosion. Many people are still working on the improvement of the Big-Bang model or the construction of other totally new cosmological models. Recently, a novel cosmological model based on Buddhist philosophy was proposed by the present author and it seems conceptually clear and logically consistent and it can explain many phenomena which belongs to the frontier problems of modern sciences. The purpose of this paper is to explain that BCM follows the general requirement of scientific criteria. Thus, the paper addresses the nature of some fundamental questions such as what science is, how to judge whether a theory is scientific or not, what do you expect from a cosmological model and how to establish a scientific cosmological model.
\end{abstract}

Keywords: Universe; World; Cosmology; The Big-Bang Cosmological Model (BBCM); The Buddhist Cosmological Model (BCM); Science; Scientific criteria

\section{Introduction}

Where did we come from? Where should we go after dying? Who am I? When did the universe begin? How big is it? Will it end? Does the universe operate according to some laws or randomly? These and many other similar questions have been explored since very ancient times by many people including scientists, philosophers, religionists, and many theories have been proposed [1-3]. Today, the consensus among scientists, astronomers and cosmologists is the Big Bang Theory also known as the Big-Bang Cosmological Model (BBCM) [3,4]. The basics of the Big Bang theory are simple. In short, the Big Bang hypothesis states that all the current and past matter in the Universe came into existence at the same time, roughly 15 billion years ago. At this time, all matter was compacted into a very small ball with infinite density and intense heat called a Singularity. Suddenly, the Singularity began expanding, and the universe as we know it began [4]. This model has been confronted to a variety of observations that allow one to reconstruct its expansion history, its thermal history and the structuration of matter. Hence, what we refer to as the BBCM today is radically different from what one may have had in mind a century ago. Even the latest version of BBCM still suffers a lot of challenges [5]. Many paradoxes are related to the use of this model [6]. Burago pointed out that at least two obvious questions exist in the Big-Bang model [7]. The first question remained as to how the matter and energy were in this superdense elementary particle? It is considered incorrect to ask, what was around this particle before the explosion and whether the universe does expand? Because space and time in the universe also arose as a result of the Big Bang. It is assumed that protons, neutrons, positrons, electrons and other longlived elementary particles formed 15 billion years ago and have reached our days unchanged. The second question arose from the insolvency of ideas about the explosion of a kind of "cosmic egg", which was the explosion of the largest nuclear bomb. This point of view boils down to the assertion that "space" exploded, and not a material object. At the same time, the authors of this idea do not bother explaining what they think is a "space" and what can explode in an empty space? Therefore, many people are still working on the improvement of the Big-Bang model or the construction of other totally new cosmological models [3].

In order to overcome these difficulties, recently, a novel cosmological model based on Buddhist philosophy was proposed by the present author [8] and from his judgement, it seems conceptually clear and logically consistent and it can explain many phenomena which belongs to the frontier problems of modern sciences [9]. The purpose of this paper is to explain that BCM can be strictly subjected to the scrutinization of scientific criteria. Thus, the paper addresses the nature of some fundamental questions such as what science is, how to 
judge whether a theory is scientific or not, what do you expect from a cosmological model and how to establish a scientific cosmological model.

\section{Science and Scientific Nature}

Now it is the time of science ruling period and it is more or the less equivalent that I am wrong if I am blamed to be unscientific [10]. Many people even believe that science represents the truth. However, if you ask someone what science is and what are his criteria to be scientific, he probably could not answer. When the present author has the chance to interview Ph.D. candidates, occasionally he asked these questions and very often the candidate could not answer. Therefore, it is not natural that everyone understands science. When they talk about scientific concepts, they do not always mean the same. So, in this section, I would like to present his view on science and its nature to be scientific.

Webster's New Collegiate Dictionary gives the definition of science as "knowledge attained through study or practice" or "knowledge covering general truths of the operation of general laws, esp. as obtained and tested through scientific method and concerned with the physical world". The same website has also provided some other common definitions of science:

1. A branch of knowledge or study dealing with a body of facts or truths systematically arranged and showing the operation of general laws: for example, mathematical science;

2. Systemic knowledge of the physical or material world gained through observations and experimentation;

3. Systematized knowledge in general;

4. Any of the branches of natural or physical sciences;

5. Knowledge of facts or principles; knowledge gained by systematic study;

6. Skill especially reflecting a precise application of facts or principle.

Personally, I do not like above definitions, especially those related to the concept "truth". I prefer the following definition:

"Science is defined as the observation, identification, description, experimental investigation, and theoretical explanation of natural phenomena" [11].

In this definition, it is first emphasized that science is a manmade language developed for communicating and understanding the observed natural phenomena among human beings. Second, every scientific theory has at least three components: axioms, laws and natural phenomena. Axioms are fundamental assumptions called hypotheses. Hypotheses do not need to be proved, but if a counter example is found against one hypothesis or a paradox is found if one accepts this hypothesis, the theory is proved to be wrong. The laws can be derived from the logical deduction from the axioms or from the logical induction from the natural phenomena. From this point we can know that scientific tool can prove the falsehood but cannot prove the axiom to be always true. Repeat of 1000 times does not guarantee that the 1001 time will repeat again. Up to now there has not been found a counter example against the axiom, it does not guarantee there will be no counter examples against the axiom in the future. So, every axiom and law used in a scientific theory is only of relative or temporary correctness and it should not be regarded as a truth if we define the truth to be a universal law. If someone takes an axiom or a law to be a truth, it is his belief rather than the scientific evidence. The attitude itself is not very scientific since scientific spirit encourages people to question every axiom or law [12]. Only when I could not find any counter examples to an axiom or a law, should I accept this axiom or law. Recently, the argument on the safety of the quantum communication is heated and some experts made the statement "The quantum communication is absolutely safe". From the point here, this statement is not scientific. Third, when one discusses the relation between science and religion, e.g., [13], one often thinks that science is based on objective evidence while religion is based on subjective belief. As a matter of fact, fundamentally speaking, the basis for us to accept a scientific theory is also a subjective belief since we cannot prove the axioms to be always true. In terms of the belief, it belongs to philosophy. So, every scientific theory is stood on the foundation of a philosophy $[14,15]$.

In order to illustrate this point, we can take the quantum mechanics as an example. The interpretation of quantum phenomena had resulted in a long debate among two camps led by Einstein and Bohr respectively and it is basically a philosophical debate [16]. The present quantum mechanics is based on the Copenhagen interpretation. It says that a measurement causes an instantaneous collapse of the wave function describing the quantum system, and the system after the collapse is random. A strong support to this random nature is the so-called Heisenberg's uncertainty principle $[17,18]$. Recently, Das explored several published proofs of uncertainty principle, including Heisenberg's and Operator theoretic, and analyzed the assumptions behind them and pointed out that uncertainty principle is a consequence of Fourier transform (FT) based on infinity assumption [19]. As infinity is not realistic and meaningful in nature, he showed that replacing infinity by any finite value changes the lower bound of the uncertainty principle to any desired accuracy number. Das further points out that uncertainty principle violates a very fundamental and well-known concept in mathematics: the infinite dimensionality property of functions over finite intervals and it cannot be a law of nature. It is impossible to prove uncertainty principle by any physical experiment [19]. Using logic and mathematics, Barukčić showed that Heisenberg's uncertainty principle leads to a contradiction and is based on a contradiction. Consequently, Heisenberg's uncertainty principle is refuted in general. Especially, Heisenberg has not refuted the principle of causality [20]. 
Albert Einstein did not believe in the idea of genuine randomness in nature and he thought that the Copenhagen interpretation is incomplete and suggests that there should be "hidden" variables responsible for random measurement results [21]. In 1935, Einstein, Podolsky and Rosen proposed a thought experiment and condensed the philosophical discussion into a physical argument [22]. In 1964, J. Bell proposed his mathematical formulation for EPR paradox [23]. He constructed a well-known inequality and showed that it was incompatible with the statistical predictions of quantum mechanics [24]. In the past decades, Bell's formulation has been frequently questioned but it is still overwhelming in the sciences of quantum information and computation. Cao and Liu studied the assumptions needed in Bell's mathematical argument and they showed the contradictions among these assumptions. Consequently, they concluded that Bell's argument for EPR paradox is illogical and Bell's inequality is trivial [25]. Recent experimental results $[26,27]$ also showed that the evolution of each completed quantum jump is continuous, coherent and deterministic. Their findings support the modern quantum trajectory theory [28]. In terms of the debate between Einstein and Bohr, the present author concluded that this controversy was created by Bohr's over claim [29]. Philosophically speaking, there is no need to make the claims that

1. there is no rule in the micro world operation and

2. there exists irreducible uncertainty or objective uncertainty.

If hidden variables related to the mind forces are included, then classical statistical mechanics can also explain the quantum phenomena [30].

In general, accuracy and correctness are always a contradiction. For the given information, the more accurate the less correct [31]. For example, if one answers the question "what is Smith's height", if he answers he is $1.75 \mathrm{~m}$, he is probably wrong and if he answers he is between $1.70 \mathrm{~m}$ to $1.80 \mathrm{~m}$, he may be certainly right. The main task of establishing a scientific theory is to maximize the accuracy for the given information under the condition of correctness. If it is incorrect, the accuracy is meaningless. It is a very interesting phenomenon we found that modern western sciences paid more attention to accuracy and they need to be updated frequently such as from Newtonian mechanics to Einstein's theory of relativity. However, ancient oriental sciences such as Chinese medicine "Huang di's inner classic - basic questions" [32], Chinese Philosophy "Lao Zi: The Book of Tao and De" [33], Buddhist Philosophy [34] were never found to be wrong although it is not very accurate for applications. Why those authors could lay a foundation to such an extent of correctness at very ancient times is worth for further study.

The philosopher of science Karl Popper proposed falsification as a criterion of demarcation to draw a sharp line between those theories that are scientific and those that are unscientific [35].
Although this criterion has not been fully agreed by many modern philosophers of science, for example, Rafe Champion criticized, "Popper's ideas have failed to convince most professional philosophers because his theory of conjectural knowledge does not even pretend to provide positively justified foundations of belief. Nobody else does better, but they keep trying, like chemists still in search of the Philosopher's Stone or physicists trying to build perpetual motion machines" [36]. Personally, I can accept his criterion, however, in different from his opinion that there exist some non-falsifiable theories, I declare that every theory is falsifiable. For example, although one may not be able to find a counter example to falsify the statement, he may be able to derive one paradox from this statement if this statement is scientifically wrong. Today I have not found the counter example does not mean tomorrow I will not be able to find. That is the nature of science. Thus, my criterion of demarcation to draw a sharp line between those theories that are scientific and those that are unscientific is dynamic. If our human beings have not falsified the hypotheses of a theory, it is still a scientific theory; otherwise if we have found a counter example or a paradox of a theory, it is unscientific. But if the application range is refined to the scope where the counter example or the paradox can be removed, it is still a scientific theory. Newtonian mechanics is a typical example and, in the future, quite a lot of modern scientific theories may be subjected to this type of revision. It is my belief that science is adequate to falsify an unscientific theory but inadequate to prove it is a truth. In that sense, Popper's falsification criterion can be used to demarcate science from pseudoscience.

Others may choose replicability (or repeatability) as a demarcation criterion of science from pseudoscience. Braude provided a detailed discussion in an Editorial and his conclusions are "that position is both shallow and confused, and the problems with it don't even have the virtue of being subtle. First, the skeptical reliance on the demarcation criterion rests on a naïve conception of the actual importance within science of experimental repeatability. Indeed, experimental repeatability plays little if any role in disciplines (including some physical sciences) whose scientific credentials are not in dispute. Second, it seriously misconstrues how the appeal to replicability works even in those physical sciences where it plays a real role. Third, the received view rests on philosophical confusions regarding the nature of similarity-in the flawed idea that there can be formal, context-independent, criteria for the similarity of two things. And fourth, it rests on confusions over the nature of human abilities generally, and, the appropriate methodologies for studying them" [12].

\section{What kind of problems need be addressed in a cosmo- logical model?}

Following Karl Popper's famous opinion, "All science is cosmology, I believe", cosmology is the foundation of all other sciences. Cosmology is intrinsically linked with mythology and 
religion as a quasi-rational elaboration of the former. Behind each cosmological model it is the philosophical belief of the proposers. It is well-known that the currently most prevailing cosmological model, the Big Bang theory, is based on materialism in which it is stated that matter is the fundamental substance in nature, and that all other things, including mental aspects and consciousness, are results of material interactions [10]. With this philosophical monism, many paradoxes can be found, and the most famous ones are Zeno's paradoxes [37]. From my point of view, the most difficult problem should be the "creator problem" [38]. How was the first matter created and where did the force come from for creating this matter? This problem also exists in the modern string theory such as what are the strings in the universe and who makes them vibrate? [39]. As a matter of fact, since the discovery of Einstein's famous equation, it has already been proved that the fundamental assumption made in materialism is wrong since matter can be transformed into energy. Schramm [40] has pointed out that very few physical theories are in such a paradoxical situation as Big-Bang cosmology which is completely based on materialism. In this monism, there are no clear definitions of matter and consciousness. In order to explain the redshift phenomenon observed, concepts such as dark matter and dark energy had to be introduced [1], but we are still unclear what they are after many decades' research $[41,42]$. Frank Wilczek [43] has optimistically told us that in theoretical physics, paradoxes are good, and paradoxes focus our attention, and make us think harder. This is also true for me why I started to doubt the materialism. Through reading, I have found many criticisms to materialism, e.g. [10,44]. Due to this reason, I has made a comparison of different philosophies and found that only Buddhist philosophy can match the logical consistency requirements of modern sciences and so I constructed a parallel cosmological model based on Buddhist philosophy (Buddhist cosmological model, BCM) in replacing the Big-Bang cosmological model (BBCM) [8] and a detailed comparison is made between BCM and BBCM [9]. I found that $\mathrm{BCM}$ can overcome all the paradoxes encountered by BBCM. It is very interesting to me that Einstein had reached the same conclusion: "The religion of the future will be cosmic religion. The religion, which is based on experience, which refuses dogmatic. If there's any religion that would cope the scientific needs, it will be Buddhism..." [13]. However, I don't think Einstein fully understood Buddhism and he was fundamentally still a believer of materialism since all the sciences he learned at that time were implicitly based on materialism. Otherwise, he should not lose the debate to Bohr in the explanation of the quantum phenomena [30]. The reason why Einstein mentioned Buddhism because he admired Spinoza. Spinoza's concept of religion is considered as pantheism. Pantheism is alien to European cultural sphere and is more appropriate to relate to Buddhism, which is not based on the concept of personal God at all. "Einstein was from the very youth inclined to question unquestionable, suspect self-evident, test trivial. His ideas on space (commensurability) and time (simultaneity), put into the formulae that will be called Special
Theory of Relativity, were fruit of some five years meditations, as recognized by Einstein himself" [45].

So, what problems should be addressed in a cosmological model? In general, any questions related to the concept of universe should be addressed. For example, when did the universe begin? How big is it? Will it end? Does the universe operate according to some laws or randomly? Where did we come from? Where should we go after dying? Who am I? In celebrating the 125th anniversary of Science, 125 big questions have been published and 25 of the 125 questions are selected to highlight based on several criteria: how fundamental they are, how broadranging, and whether their solutions will impact other scientific disciplines. The first question is what the universe is made of [46]. Now we have already known that the universe at least contains energy, matter, dark energy, dark matter, life and information. A cosmological model should answer these important questions.

\section{How to establish a scientific cosmological model?}

The first step is to make clear definitions for basic concepts. The concepts to be defined include universe, world, time, space, matter, energy, dark matter, dark energy, consciousness, mind, etc. The second step is to make fundamental hypotheses. The less the better. The third step is to derive the laws from hypotheses; and the fourth step is to use these laws to explain the observed phenomena or to predict the future phenomena. The fifth step is to revise and improve theory through application.

As is clear from section 2, every theory is based on hypotheses. Only if hypotheses are related to infinity, it could be an ultimate theory [47]. Otherwise, it could lead to a lot of origination problems. So, in BCM, the first hypothesis is basically a definition.

1. Hypothesis 1: The Universe is of infinite nature both in time and space. It can be divided into infinite number of worlds in space. World is of finite nature both in time and space. Each world is cyclically operated according to the process of formation, the steady state, deterioration and explosion to emptiness.

The relationship between Universe and world can be expressed by the following equation:

$$
\text { Universe }=\sum_{i=1}^{\infty} \text { World }_{i}
$$

In BCM, the Big Bang is the origin of the world but not the universe. Using this definition, the awkward question of the origin of the universe can be avoided and the origin of each world can easily be explained. In this hypothesis, we have used the concepts of space and time. In BCM, we do not provide any new definitions for space and time. A quote of Kant is: "Space and time are the framework within which the mind is constrained to construct its experience of reality" [14]. From this hypothesis, what we have observed is the phenomena in the world we are living, and the world is in this cycle. So, both time and space should be of finite nature for a world. That should be the socalled boundedness law and finite time law mentioned in [19]. It 
is not appropriate to apply these two laws to the Universe as the author indicated.

This assumption can combine the main points presented in the infinite universe model [47], cyclically universe model [48] and the many-worlds interpretation model [49].

2. Hypothesis 2: The essence of the universe is energy. There are two types of energy, material energy and life energy. Life energy is the source of all forces in the universe and it is responsible for all the changes and movements observed by our human beings. Life energy has the capability to accumulate the material energy into matter and to decompose matter into material energy. An individual life is formed if life energy is combined with matter (body). Each life is reincarnated in the universe from birth to death.

In this hypothesis, the following concepts are involved, energy, matter and life. In different from materialist philosophy who assumed that the basic elements are the essence of universe, we assumed energy is the essence of the universe. For energy, there is no time, space, volume or other measurable properties. This can eliminate the origination problem for basic elements. Matter is defined to have volume and mass. Mass could be static or dynamic. Energy has two types; one is material energy which can be accumulated to be matter and another is life energy and we interpret it to be the mind. It is also speculated that this life energy corresponds to the dark energy defined in modern physics. The reason we interpret the life energy as mind follows the opinion of René Descartes. The discourse on consciousness has been hugely influenced by René Descartes, the French philosopher who in the mid-17th century declared that body and mind are made of different stuff entirely. It must be so, Descartes concluded, because the body exists in both time and space, whereas the mind has no spatial dimension [50]. Matter has two types; one is what we can feel and the other is what we cannot feel (corresponding to the dark matter defined in modern physics). By adopting this hypothesis, the origin of matter is answered, and all matter are created by mind by accumulating the material energy in a world and the mind is also able to decompose any matter into material energy. Therefore, there are no basic elements in BCM. This may provide the physical explanation to string theory [39]. String theory intends to provide a unified description of gravity and particle physics that describes all fundamental forces and forms of matter.

Based on Buddhism the human's consciousness includes 8 types, they are consciousness at eyes, ears, nose, tongue, body and brain, the manas consciousness, and finally the alaya consciousness. When one is dying, the consciousness at eyes, ears, nose, tongue, body and brain will be lost. The function of the seventh consciousness whose formal name is called the manas consciousness, is the bridge between the former six consciousness with the eighth whose formal name is called the alaya consciousness. Only the alaya consciousness will continue to exist and it is the source of life forces and it stores all the karmas in the previous life history. For simplicity, this alaya consciousness is defined as mind in BCM. The dying process is a process of the separation of the mind from the body. For the detailed theory of Buddhism, one can refer to the reference [51]. So, mind is the energy while other seven consciousnesses are related to the body. In BCM, both plants and animals also have mind and some types of consciousnesses but may not have as many as human beings.

3. Hypothesis 3: Everything in the world and each individual life is operated according to the Causal-Effect law.

It is emphasized in BCM that since we can only observe the world we are living, so every theory should only be established to system problems in the world rather than those in the universe. Since world has boundary and finite space, each cycle is of finite time, then we take the belief that world is operated with rules and our human beings can reveal these rules. The concept of the world is similar as a system, we can call everything a world, the largest world is the one which can be observed by human beings. It is certainly dynamic and as the technology progresses, the boundary moves outwards, but its nature to be finite will never change. It is the fundamental assumption that the Universe is infinite.

For any lifeless object from a particle to a star in the world we are living, no matter whether we can see (explicit matter) or not (dark matter), it will experience the cycle of formation, the steady state, deterioration and destroy and for each individual life, no matter whether we can see (human beings and animals) or not (other four types of lives), she/he will be reincarnated within the six types of lives in the universe [8]. Here it must be pointed out that according to the causal-effect law, reincarnation of my live is not confined to this world I am living, but in the whole universe. Even for the alaya consciousness only life in the Heaven, she/he will also have a life span and can be reincarnated into other five types with a body in the universe, not necessarily in the same world as he/she lived in a previous life. Reincarnation of all lives is a law of nature [52]. So, in this BCM, parents provide only the bodies to their children and not the life. We are very soon reached a stage that we can clone our bodies, but the essence of life does not change, and any lives produced this way should have the same rights as us.

These three hypotheses are adequate to explain all the anomalous phenomena reported in the book [53] such as Near-Death Experience (NDE), Out of Body Experience (OBE), mediumship and children claiming past-life memories etc. From these three hypotheses, many laws can be derived, and this is certainly a tremendous work and will be discussed in subsequent papers. Here only one important law is emphasized.

1. Law 1: There never exists any closed or isolated system except the Universe.

It is well-known that traditionally we have classified a thermodynamic system of three types, a closed system, an isolated 
system and an open system [54]. A closed system can exchange energy (as heat or work) but not matter, with its surroundings. An isolated system cannot exchange any heat, work, or matter with the surroundings, while an open system can exchange energy and matter. With these definitions, the dark matter, the dark energy and information which might be important to the system state are not included. In this paper, information is defined as the useful messages used for communication among living creatures. It includes data and knowledge and it is neither energy nor matter and it can be transmitted through energy and matter. Information, the dark matter and the dark energy could also exert influence on the system behavior.

From hypotheses 1 and 2, one can be aware that there never exists any closed or isolated system in a world. Since Universe is infinite, it is meaningless to discuss any nature of Universe, i.e., it could be the same if we assume Universe to be a closed system or an isolated system or an open system. Since all the systems our human beings faced should be in a world, no matter how big it is such as the Milky Way or even larger Galaxy system, it is an open system in nature since we could not prevent the exchange of information, energy and even dark matter at the system boundary. Therefore, the assumption of a closed system or an isolated system is purely mathematical and does not represent the reality. Thus, all the laws derived by employing this assumption should be subjected to the scrutinization to identify their application ranges.

\section{Summary and Conclusion}

The cosmological model is very important, and it is the basis for other sciences. The currently most prevailing cosmological model for the observable universe, the BBCM, still exists many un-answered questions. In order to overcome these problems, a novel cosmological model based on Buddhist philosophy (BCM) was proposed by the present author and it seems conceptually clear and logically consistent and it can explain many phenomena which belongs to the frontier problems of modern sciences $[8,9]$. However, whether the BCM is scientific or unscientific is the most important concern for readers. So, the main purpose of this paper is to explain that BCM follows the general requirement of scientific criteria. Thus, the paper addressed the nature of some fundamental questions such as what science is, how to judge whether a theory is scientific or not, what do you expect from a cosmological model and how to establish a scientific cosmological model.

From these discussions, the following conclusions can be drawn:

1. The nature of science is to falsify the wrong but unable to prove a universal truth. All the laws are of relative correctness and will be of specific application range.

2. With a definition of infinite Universe and finite worlds, the essence of Universe is energy and there are two types of energy, the origination problem of the Universe can be avoided, and the origination of worlds can easily be explained by the Universe mind.

3. Irreducible uncertainty only exists with the concept of Universe and in each world the operation will follow the causal-effect law. This law can be revealed by human beings. Thus, all the uncertainties related to the world problems can be reducible as the information and knowledge increases.

4. For all the systems in a world, it should be an open system. All the laws derived by employing closed system or isolated system assumption should be subjected to the scrutinization to identify their application ranges.

Of course, the perfection of this BCM is a tremendous task and this paper just summarizes some important points and many points need to be proved more strictly with logic and mathematics. This will be done subsequently.

\section{Acknowledgement}

This work was supported by the State Key Program of National Natural Science of China "Structural Reliability Analysis on the Spherical Hull of Deepsea Manned Submersibles" (Grant No. 51439004), the General Program of National Natural Science of China "A study on the water absorption property of the buoyancy material for the full ocean depth manned submersible" (Grant No. 51879157), the "Construction of a Leading Innovation Team" project by the Hangzhou Municipal government, the Startup funding of New-joined PI of Westlake University with grant number (041030150118).

\section{References}

1. Wilczek F (1982) Old and new relics in cosmology (A Review). Proc Natl Acad Sci USA 79(10): 3376-3379.

2. Greenstein JL (1993) An ancient revisits cosmology. Proc Natl Acad Sci USA 90(11): 4878-4881.

3. Joyce A, Jain B, Khoury J, Trodden M (2015) Beyond the cosmological standard model. Physics Reports 568: 1-98

4. Uzan JP (2015) The Big-Bang Theory: Construction, Evolution and Status. Seminaire Poincare, pp. 1-69.

5. Steigman G (1993) Challenges to the standard model of Big Bang nucleosynthesis. Proc Natl Acad Sci USA 90(11): 4779-4781.

6. Clark M (2007) Paradoxes from A to Z. ( $2^{\text {nd }}$ edn), Taylor \& Francis e-Library, UK.

7. Burago SG (2017) About the theory of the Big Bang, The General Science Journal. Astrophysics pp. 1-7.

8. Cui WC (2019) On A Logically Consistent Cosmological Model Based on Buddhist Philosophy. Ann Soc Sci Manage Stud 3(1): 555605.

9. Cui WC (2019) A Comparison of BCM with BBCM. Ann Soc Sci Manage Stud 3(3): 555612.

10. Sheldrake R (2012) The Science Delusion---Freeing the Spirit of Enquiry. Coronet, An imprint of Hodder \& Stoughton, A Hachette UK company.

11. Lyndsay TW (2009) Definition of Science.

12. Braude SE (2018) Editorial. Journal of Scientific Exploration 32(2): 255-264. 
13. Einstein A (1930) "Religion and Science", New York Times Magazine, cited from Einstein A (1954), "Ideas and Opinions", Crown Publishers, New York, USA.

14. Kant I (2002) Metaphysical Foundations of Natural Science, in Theoretical Philosophy after 1781, edited by Henry A, Peter $\mathrm{H}$ translated by Michael F, Cambridge University Press, Cambridge, UK.

15. Laplane L, Mantovanic P, Adolphs R, Chang H, Mantovani A, et al. (2019) Why science needs philosophy. Proc Natl Acad Sci USA 116(10): 39483952.

16. Whitaker A (2006) Einstein, Bohr and the Quantum Dilemma - From Quantum Theory to Quantum Information $2^{\text {nd }}$ (edn) Paperback Cambridge University Press, UK.

17. Heisenberg W (1927) The physical content of quantum kinematics and mechanics. Z Physik 43: 172-98. (Translation into English by JAW, WHZ (1981) Available in the book Quantum theory and measurement (1983) Wheeler JA, Zurek WH pp.62-84, Princeton university press, NJ, USA).

18. Heisenberg W (1930) The physical principles of the quantum theory Translated in English, Eckart C, Hoyt FC, Dover publications, University of Chicago, USA

19. Das S (2013) Assumptions in Quantum Mechanics. International Journal of Theoretical and Mathematical Physics 3(2): 53-68.

20. Barukčić I (2016) Anti-Heisenberg- The End of Heisenberg's Uncertainty Principle. Journal of Applied Mathematics and Physics 4(5): 881-887.

21. Bohm D (1952) A Suggested Interpretation of the Quantum Theory in Terms of "Hidden" Variables I\&II. Physical Review 85(2): 166-193.

22. Einstein A, Podolsky B, Rosen N (1935) Can quantum-mechanical description of physical reality be considered complete. Phys Rev 47(10): 777-780.

23. Bell JS (1964) On the Einstein-Podolsky-Rosen paradox. Physics 1(3): 195-200.

24. Bell JS (1966) On the problem of hidden variables in quantum mechanics. Rev Mod Phys 38(3): 447-452.

25. Cao ZJ, Liu LH (2018) Remarks on Bell's Inequality. Cryptology ePrint Archive, pp. 1-10.

26. Minev ZK, Mundhada SO, Shankar S, Reinhold P, Gutierrez-Jauregui R, et al. (2019) To catch and reverse a quantum jump mid-flight. Nature 570: 200-204.

27. Minev ZK (2018) Catching and Reversing a Quantum Jump Mid-Flight. PhD thesis, Yale Univ, USA.

28. Hiley BJ, Reeth PV (2018) Quantum Trajectories: Real or Surreal? Entropy 20(353): 1-18.

29. Cui WC (2019) A Simple Idea on the Unification of Einstein-Bohr Controversy. Ann Soc Sci Manage Stud 2(5): 555597.

30. Cui WC, Kang BL (2008) Examining the debate between Einstein and Bohr based on Buddhist philosophy. Journal of Chinese Social Sciences 55(8): 1-9.

31. Cui WC, Blockley DI (1990) Interval probability theory for evidential support. Int J of Intelligent Systems 5(2): 183-190.

32. Unschuld PU (2011) Huang di nei jing su wen [electronic resource]: an annotated translation of Huang di's inner classic - basic questions Berkeley: University of California Press, USA.
33. Gu Z (1995) Lao Zi: The Book of Tao and De. Beijing University Press, China.

34. Laumakis SJ (2008) An Introduction to Buddhist Philosophy. Cambridge University Press, New York, USA.

35. Popper K (1963) Conjectures and Refutations: The Growth of Scientific Knowledge (2002 edn), London: Routledge, UK.

36. Champion R (2013) Reason and Imagination (Critical Rationalist Papers Book 2). ASIN B00CGETUCM.

37. Salmon WC (1970) Zeno's Paradoxes. Bobbs-Merrill, New York, USA.

38. Sarfati JD (1998) If God Created the Universe, Then Who Created God? CEN Tech J 12(1): 20-22.

39. Zwiebach B (2004) A First Course in String Theory, Cambridge University Press, New York, USA.

40. Schramm DN (1998) The age of the universe, dark matter, and structure formation. Proc Natl Acad Sci USA 95: 1

41. Bahcall NA (2015) Dark matter universe. Proc Natl Acad Sci USA 112(40): 12243-12245.

42. Arun K, Gudennavar SB, Sivaram C (2017) Dark matter, dark energy, and alternate models: a review. Advances in Space Research 60: 166186.

43. Wilczek F (2005) Asymptotic freedom: From paradox to paradigm Proc Natl Acad Sci USA 102(24): 8403-8413.

44. Tart CT (2009) The End of Materialism: How Evidence of the Paranormal Is Bringing Science and Spirit Together, New Harbinger Publications, Inc, Oakland, USA.

45. Dokovic V, Grujic P (2007) Albert Einstein, Cosmos and Religion. Serb Astron J 174: 61-72.

46. Seife C (2005) What Is the Universe Made Of? Science 309(5731): 78.

47. Borchardt G (2017) Infinite universe theory (Version 20180525): Berkeley, CA, Progressive Science Institute, USA.

48. Steinhardt PJ, Turok N (2002) A Cyclic Model of the Universe. Science 296(5572): 1436-1439.

49. DeWitt BS, Graham RN (Eds.) (1973) The Many-Worlds Interpretation of Quantum Mechanics, Princeton Series in Physics, Princeton University Press, USA.

50. Miller G (2005) What Is the Biological Basis of Consciousness? Science 309(5731): 79.

51. Harvey P (2013) An Introduction to Buddhism, Teachings, History and Practices, ( $2^{\text {nd }}$ edn), Cambridge University Press, Cambridge, New York, Melbourne, Madrid, Cape Town, Singapore, São Paulo, Delhi, Mexico City.

52. Das S (2013) Reincarnation-a Law of Nature. International Journal of Modern Engineering Research (IJMER) 3(2): 1192-1211.

53. Moreira-Almeida A, Santos FS (Eds.) (2012) Exploring Frontiers of the Mind-Brain Relationship, Springer, New York, Dordrecht Heidelberg, London, UK.

54. von Bertalanffy, Ludwig (1968) General System theory: Foundations, Development, Applications, New York: George Braziller. 


\section{Your next submission with Juniper Publishers} will reach you the below assets

- Quality Editorial service

- Swift Peer Review

- Reprints availability

- E-prints Service

- Manuscript Podcast for convenient understanding

- Global attainment for your research

- Manuscript accessibility in different formats ( Pdf, E-pub, Full Text, Audio)

- Unceasing customer service

Track the below URL for one-step submission https://juniperpublishers.com/online-submission.php 